\title{
Ultrasound-guided lateral-medial transmuscular quadratus lumborum block for analgesia following anterior iliac crest bone graft harvesting: a clinical and anatomical study Analgésie réalisée grâce à un bloc transmusculaire latéro-médial échoguidé du carré des lombes suite à un prélèvement de l'os de la crête iliaque antérieure pour greffe osseuse : une étude clinique et anatomique
}

\author{
Rakesh V. Sondekoppam, MD - Vivian Ip, MBCHB, FRCA · David F. Johnston, MB, \\ BCh, FRCA EDRA, PGCert • Vishal Uppal, MD, FRCA · Marjorie Johnson, BSc, PhD • \\ Sugantha Ganapathy, MD, FRCPC • Ban C. H. Tsui, MD, FRCPC
}

Received: 22 June 2017/Revised: 18 October 2017 / Accepted: 18 October 2017/Published online: 21 November 2017 (C) Canadian Anesthesiologists' Society 2017

\begin{abstract}
Background The anterior iliac crest (AIC) is one of the most common sites for harvesting autologous bone, but the associated postoperative pain can result in significant morbidity. Recently, the transmuscular quadratus lumborum block (TQL) has been described to anesthetize
\end{abstract}

R. V. Sondekoppam, MD · V. Ip, MBCHB, FRCA Department of Anesthesia, University of Alberta, Edmonton, $\mathrm{AB}$, Canada

D. F. Johnston, MB, BCh, FRCA EDRA, PGCert .

S. Ganapathy, MD, FRCPC

Department of Anesthesia, University Hospital, London Health

Sciences Centre, Windermere Road, London, ON, Canada

V. Uppal, MD, FRCA

Department of Anesthesia, University of Dalhousie, Halifax, NS, Canada

M. Johnson, BSc, PhD

Department of Anatomy and Cell Biology, University of

Western Ontario, London, ON, Canada

B. C. H. Tsui, MD, FRCPC

Department of Anesthesiology, Perioperative, and Pain Medicine, Stanford University Medical Center, Palo Alto, CA, USA

B. C. H. Tsui, MD, FRCPC ( $\square)$

Department of Anesthesiology, Perioperative and Pain Medicine, Stanford University School of Medicine, 300 Pasteur Drive, H3582, Stanford, CA 94305-5640, USA

e-mail: bantsui@stanford.edu the thoraco-lumbar nerves. This study utilizes a combination of cadaveric models and clinical case studies to evaluate the dermatomal coverage and analgesic utility of TQL for AIC bone graft donor site analgesia.

Methods Ten ultrasound-guided TQL injections were performed in five cadaver specimens using a lateral-tomedial transmuscular approach. Twenty $m L$ of $0.5 \%$ methylcellulose was injected on each side after ultrasound confirmation of the needle tip ventral to the quadratus lumborum muscle (QLM). Cranio-caudal and medial-lateral extent of the dye spread in relation to musculoskeletal anatomy and direct staining of the thoraco-lumbar nerves were recorded. Following the anatomical findings, continuous catheter TQL blocks were performed in four patients undergoing ankle surgery with autologous AIC bone graft. The dermatomal anesthesia and postoperative analgesic consumption were recorded.

Results In the anatomical component of the study, 9/10 specimens showed a lateral spread anterior to the transversalis fascia and medially between the QLM and psoas major muscle. Direct staining of the branches of the T12, L1, and L2 nerves was noted ventral to the QLM, while variable staining of the T9-T11 nerves was seen laterally in the transversus abdominis plane and the transversalis fascia. The vertical spread of injectate anterior to the QLM was T12 to the iliac crest $(n=5)$ $10)$ and $L 1$ to the iliac crest $(n=4 / 10)$. In the four patients 
who received $T Q L$, the T9-L2 dermatomal anesthesia correlated with the injectate spread seen in the cadavers and provided effective analgesia at the bone graft donor site.

Conclusion Ultrasound-guided TQL injections consistently cover the thoraco-lumbar innervation relevant to the AIC graft donor site. The injectate spread seen in anatomical dissections correlated with the dermatomal anesthesia clinically. The TQL has the potential to provide reliable analgesia for patients undergoing AIC bone graft harvesting.

\section{Résumé}

Contexte La crête iliaque antérieure est l'une des zones les plus fréquemment utilisées pour le prélèvement osseux autologue, mais la douleur postopératoire associée peut entraîner une importante morbidité. On a récemment décrit le bloc transmusculaire du carré des lombes (quadratus lumborum) pour l'anesthésie des nerfs thoraco-lombaires. Cette étude se fonde sur une combinaison de modèles cadavériques et d'études de cas cliniques afin d'évaluer l'étendue des dermatomes couverts et l'utilité analgésique d'un bloc transmusculaire du carré des lombes pour l'analgésie de la zone donneuse de greffe osseuse de la crête iliaque antérieure.

Méthode Dix injections transmusculaires échoguidées au niveau du carré des lombes ont été réalisées sur cinq spécimens cadavériques en utilisant une approche transmusculaire de latérale vers médiale. Vingt $m L$ de méthylcellulose 0,5\% ont été injectés de chaque côté après confirmation par échoguidage de la position ventrale de la pointe de l'aiguille par rapport au muscle du carré des lombes. La diffusion cranio-caudale et médiale-latérale de l'agent de contraste par rapport à l'anatomie musculo-squelettique et la coloration directe des nerfs thoraco-lombaires ont été évaluées. Après cette étude anatomique, des blocs transmusculaires continus du carré des lombes par cathéter ont été réalisés chez quatre patients subissant une chirurgie de la cheville avec greffe osseuse autologue de la crête iliaque antérieure. L'anesthésie dermatomale et la consommation analgésique postopératoire ont été documentées.

Résultats Dans le volet anatomique de notre étude, une dissémination latérale antérieure au fascia transversalis et médiale entre le muscle du carré des lombes et le grand psoas a été observée sur 9 des 10 spécimens cadavériques. Une coloration directe des branches des nerfs T12, L1 et L2 a été notée du côté ventral du muscle du carré des lombes, alors qu'une coloration variable des nerfs T9-T11 a été observée latéralement dans le plan abdominal transverse et le fascia transversalis. La dissémination verticale du produit d'injection du côté antérieur du muscle du carré des lombes allait de T12 à la crête iliaque $(n=5 /$
10) et de L1 à la crête iliaque $(n=4 / 10)$. Chez, les quatre patients ayant reçu un bloc transmusculaire du carré des lombes, l'anesthésie dermatomale T9-L2 se corrélait à la diffusion du produit d'injection observée chez les cadavres et a procuré une analgésie efficace du site donneur de greffe osseuse.

Conclusion Les injections transmusculaires échoguidées du carré des lombes couvrent l'innervation thoraco-lombaire pertinente à la zone donneuse de greffe de la crête iliaque antérieure. La diffusion du produit d'injection observée dans les dissections anatomiques correspondait à l'anesthésie dermatomale clinique. Le bloc transmusculaire du carré des lombes pourrait par conséquent procurer une analgésie fiable aux patients subissant une greffe osseuse de la crête iliaque antérieure.

The anterior iliac crest (AIC) is an important source of autologous bone graft due to its high content of corticocancellous bone. ${ }^{1}$ Although autologous bone graft from the anterior iliac crest is known to provide the best outcomes because of its osteo-conductive and osteo-inductive properties, the postoperative morbidity due to associated pain is a significant issue. ${ }^{2,3}$ Moderate to severe postoperative donor site pain can lead to delayed ambulation $^{4}$ and may persist as chronic post-surgical pain. ${ }^{5}$

The periosteum of the AIC is innervated by the nerves supplying the muscles attached to it and mainly includes the subcostal, ilioinguinal, and iliohypogastric nerves. ${ }^{6,7}$ The lateral femoral cutaneous nerve (L2,3) supplies the skin overlying the antero-lateral thigh and may contribute to the innervation. All the above nerves travel in close proximity to the ventral surface of the quadratus lumborum muscle (QLM) before their subsequent course in the abdominal wall. There has been an armamentarium of intermuscular plane blocks described in recent years targeting the thoraco-lumbar innervation as they pass through the thoraco-lumbar fascia. ${ }^{8}$ The trans-muscular quadratus lumborum block (TQL) ${ }^{9}$ is of particular interest for iliac crest donor site analgesia as all three nerves supplying the anterior iliac crest region travel ventral to the QLM where they can be potentially anesthetized.

Since there are different approaches to the transmuscular quadratus lumborum block, and the spread of the injectate following these injections is still unclear, we decided to examine the anatomical basis and clinical utility of a lateral to medial approach for TQL relevant for anterior iliac crest bone graft analgesia. For the purpose of our study, cadaveric TQL injections were used, and the cranio-caudal, medio-lateral extent and consistency of spread were delineated with their relation to the thoraco- 
lumbar nerves. The aim of our study was to determine from the cadaver study whether a TQL performed at the level of the L3 lumbar vertebra consistently covers the innervation of the anterior iliac crest including the subcostal, iliohypogastric, ilioinguinal, and lateral femoral cutaneous nerves. A preliminary clinical correlation of the anatomical findings in terms of dermatomal anesthesia and the analgesic effectiveness following TQL in patients undergoing AIC bone graft harvesting using similar injectate volumes was also made.

\section{Methods}

Cadaveric specimens

Five un-embalmed cadaveric specimens (Table 1) used for the anatomical component of the study were freshly thawed to room temperature over $48 \mathrm{hr}$. All cadaveric data were obtained and used in accordance with the Anatomy Act of Ontario and Western's Committee for Cadaveric Use in Research (approval \#12052014). After excluding any anatomical deformity or evidence of scars over the trunk, the cadavers were placed in the prone position for the performance of bilateral ultrasound-guided TQL at the level of the L3 transverse process.

\section{Technique of TQL injections}

All injections were performed by two anesthesiologists familiar with the techniques (R.S. and D.J.). A low frequency curvilinear 2-5 Mhz transducer (M-Turbo Sonosite, Bothell, WA, USA) was placed in a para-sagittal plane over the sacrum and the transducer moved cephalad until the lamina of the L3 vertebra was identified. Following this, the transducer was moved laterally until the L 3 transverse process was identified and the transducer was rotated by $90^{\circ}$ to obtain a transverse view of the L3 transverse process. On moving the probe laterally, the quadratus lumborum muscle could be seen attaching to the tip of the transverse process. At this level, the transverse process of the lumbar vertebrae, erector spinae muscle, psoas major muscle, and quadratus lumborum can be identified as a shamrock sign. Further scanning in this plane showed the lateral edge of the QLM, the transversalis fascia plane (TFP), and subsequently the transversus abdominis plane (TAP). After identifying the relevant anatomy, a 17G 100-mm Tuohy needle was inserted in-plane in a lateral to medial direction using ultrasound and hydrodissection techniques until the needle tip was seen to advance through the QLM and the anterior layer of the quadratus lumborum fascia. The endpoint of needle insertion was the visibility of the D5W injectate ventral to the quadratus lumborum muscle on ultrasound. After ensuring the needle tip position ventral to the QLM using 1-2 $\mathrm{mL}$ of D5W, the study injectate containing $20 \mathrm{~mL}$ of $0.5 \%$ methylcellulose with 0.5 $\mathrm{mL}$ of India ink was injected at a rate of $2 \mathrm{~mL} \cdot \mathrm{sec}^{-1}$ while observing its spread under ultrasound (Fig. 1).

\section{Anatomical dissections}

After performing the injections bilaterally, each torso specimen remained in the prone position for at least $45 \mathrm{~min}$ before the anatomical dissections were performed. All dissections were performed by an anatomist involved in the study (M.J.) but not involved in the injection technique. The skin was incised vertically along the spinous processes from thoracic vertebrae T11 to the iliac crest. Two horizontal incisions were made perpendicular to the spine at the $\mathrm{T} 11$ and iliac crest to reflect the skin laterally. The lumbar fascia, latissimus dorsi, and external oblique muscles were identified, following which the latissimus dorsi was released from its medial and inferior attachment with the thoracolumbar fascia and reflected laterally. The external oblique and internal oblique were reflected posteriorly to reveal the TAP plane and the aponeurosis of the transversus abdominis muscle. Any nerves entering the TAP plane after piercing this aponeurosis were identified using the 12 th rib as a landmark and observed for staining. The transversalis fascia was explored until the oblique lateral border of the QLM was identified. The quadratus lumborum was observed for the extent of dye spread external to its investing thoracolumbar fascia and within the muscle. After dissecting each layer of the thoraco-lumbar fascia, the medio-lateral and cranio-caudad extent of dye spread was documented in relation to the thoraco-lumbar muscles and the bony landmarks. To prevent seepage of the injectate into

Table 1 Cadaver specimen details

\begin{tabular}{llll}
\hline $\begin{array}{l}\text { Cadaver } \\
\text { number }\end{array}$ & Age & Gender & Cause of death and past medical history \\
\hline 1 & 41 & Female & Metastatic gastrointestinal (GI) neuroendocrine tumor, renal failure, upper GI bleeding \\
2 & 88 & Male & Metastatic adenocarcinoma prostate \\
3 & 92 & Male & Cardiorespiratory arrest, chronic obstructive pulmonary disease (COPD), cerebrovascular disease, hypertension, \\
& 95 & Female & Rupture of abdominal aortic aneurysm, cellulitis, dementia \\
4 & 86 & Female & End-stage COPD, non-ST elevation myocardial infarction, hypertension, peripheral vascular disease \\
5 & & &
\end{tabular}


Fig. 1 Ultrasound-guided low transmuscular quadratus lumborum block injection at L2L3 level

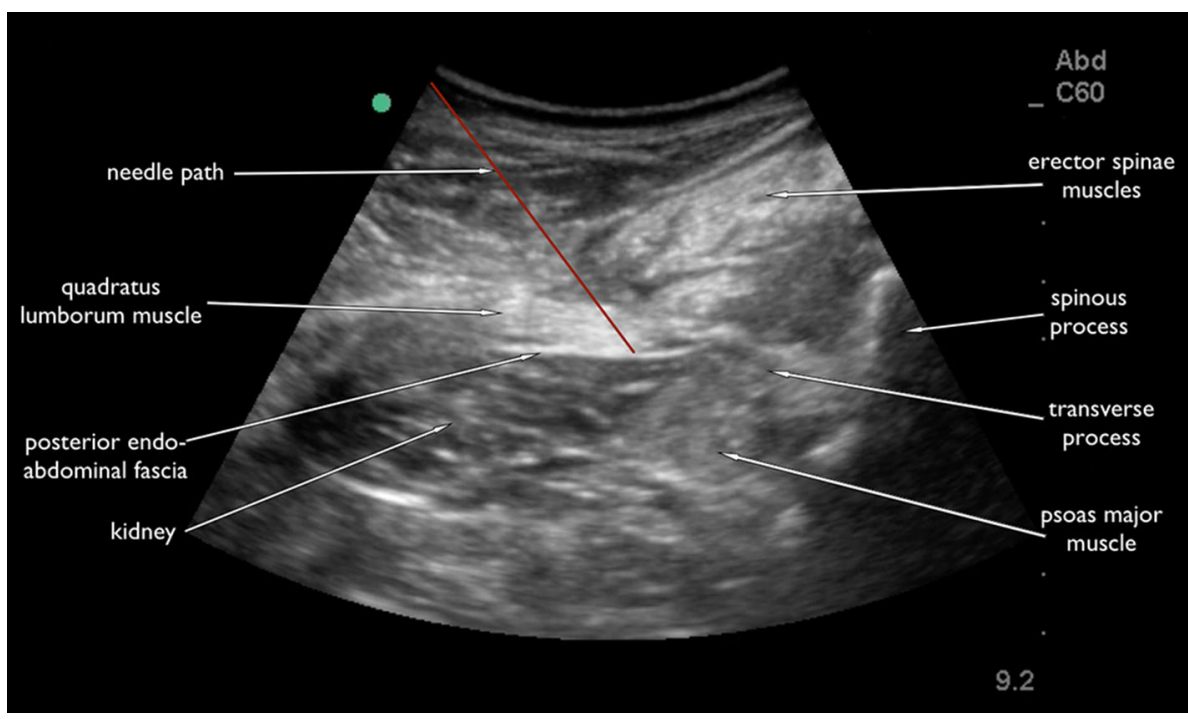

different layers during dissection, any excessive dye was dabbed after each layer of dissection. The penetration of QLM by the injectate needle was established using the dye-stained needle puncture at the posterior aspect of the muscle. The quadratus lumborum was then excised to allow visualization of the cranio-caudal extent of dye spread in relation to the QLM and its attachment to the transverse processes of the lumbar vertebra and the lower two ribs. Further, the medial extent of the dye spread towards the psoas major muscle and the paravertebral gutter was also explored. Whether any injectate was present deeper to the posterior endo-abdominal (renal) fascia covering the perinephric fat was also explored.

The cranio-caudal extent of dye spread in numbers (and percentage) has been presented in relation to the last two ribs, the transverse processes of the lumbar vertebra, and the iliac crest. The medio-lateral extent of the dye spread has been presented in relation to the thoracic paravertebral space, the psoas major muscle, the ventral surface of the QLM, the TFP, and the TAP plane.

\section{Clinical correlation of dermatomal anesthesia}

To correlate the dermatomal coverage seen with the anatomical component of our study, perioperative data from four patients undergoing ankle fusion or subtalar fusion with an iliac crest bone graft were included for the study. All patients gave written, informed consent for this case series. Preoperatively, an ultrasound-guided popliteal block was performed in a lateral decubitus position with $20-25 \mathrm{~mL}$ of local anesthetic $(0.25 \%$ bupivacaine and $1 \%$ ropivacaine, $1: 1$ ratio). In the lateral decubitus position, the TQL continuous catheter was inserted under ultrasound guidance using the same technique as described for cadaveric injections (see above) using a $21 \mathrm{G}$ 75-mm-long catheter-over-needle assembly (E-cath, Pajunk®, GmbH
Medizintechnologie, Geisingen, Germany). Twenty $\mathrm{mL}$ of local anesthetic mixture $(0.25 \%$ bupivacaine and $1 \%$ ropivacaine, 1:1) was injected into this plane after successful ultrasound confirmation of the needle tip ventral to the QLM. The needle was then withdrawn leaving the outer catheter in situ. The inner catheter was then inserted within the outer catheter. One to $2 \mathrm{~mL}$ of $5 \%$ dextrose was used to confirm the final catheter tip position under ultrasound. The catheter was then secured in place at the patient's back, well away from the sterile surgical field. The patients were then positioned supine for the ultrasound-guided saphenous nerve block with 5-10 mL of the same mixture of local anesthetic as described above. All blocks were tested using cold swabs for dermatomal anesthesia extent at $30 \mathrm{~min}$ following the block, in the postanesthesia recovery unit, and once a day in the wards.

All patients received general anesthesia as per the discretion of the anesthesiologist in the operating room. In the recovery area, the patients were assessed for resting and dynamic pain scores using a numerical rating score (0-10 scale) before discharge to the ward. All patients received intermittent boluses of $15 \mathrm{~mL}$ of $0.2 \%$ ropivacaine every 2 hr without any baseline infusion via a programmable pump through the TQL catheter. As for the popliteal catheter, intermittent boluses of $6-8 \mathrm{~mL}$ of $0.2 \%$ ropivacaine were administered every hour via the pump.

\section{Results}

Anatomical component

The QLM was successfully visualized in all the specimens bilaterally and the TQL injections were performed bilaterally in all five cadavers (Table 1). The level of 
Table 2 Spread of injectate following transmuscular quadratus lumborum block (TQL) injections at the level of L3 transverse process

\begin{tabular}{|c|c|c|c|c|c|c|c|c|c|c|c|}
\hline \multicolumn{2}{|c|}{ Cadaver } & \multicolumn{3}{|c|}{ Cephalad } & \multicolumn{2}{|c|}{ Caudad } & \multicolumn{2}{|c|}{ Medial } & \multicolumn{2}{|c|}{ Lateral } & \multirow{2}{*}{$\begin{array}{l}\text { Successful injections } \\
\text { Dye in QLF }\end{array}$} \\
\hline No. & Side & $\mathrm{T} 12$ & L1 & L2 & L4 & L5 & $\mathrm{PF}$ & PVS & TFP & TAP & \\
\hline \multirow[t]{2}{*}{1} & Left & - & $\sqrt{ }$ & - & $\sqrt{ }$ & - & - & - & $\sqrt{ }$ & - & $\sqrt{ }$ \\
\hline & Right & - & $\sqrt{ }$ & - & $\sqrt{ }$ & - & $\sqrt{ }$ & - & $\sqrt{ }$ & $\sqrt{ }$ & $\sqrt{ }$ \\
\hline \multirow[t]{2}{*}{2} & Left & - & - & - & - & - & - & - & - & - & - \\
\hline & Right & $\sqrt{ }$ & - & - & $\sqrt{ }$ & - & $\sqrt{ }$ & - & $\sqrt{ }$ & - & $\sqrt{ }$ \\
\hline \multirow[t]{2}{*}{3} & Left & $\sqrt{ }$ & - & - & $\sqrt{ }$ & - & - & - & $\sqrt{ }$ & $\sqrt{ }$ & $\sqrt{ }$ \\
\hline & Right & - & $\sqrt{ }$ & - & - & $\sqrt{ }$ & $\sqrt{ }$ & $\sqrt{ }$ & $\sqrt{ }$ & $\sqrt{ }$ & $\sqrt{ }$ \\
\hline \multirow[t]{2}{*}{4} & Left & - & - & $\sqrt{ }$ & $\sqrt{ }$ & - & $\sqrt{ }$ & - & $\sqrt{ }$ & - & $\sqrt{ }$ \\
\hline & Right & $\sqrt{ }$ & - & - & $\sqrt{ }$ & - & $\sqrt{ }$ & $\sqrt{ }$ & $\sqrt{ }$ & - & $\sqrt{ }$ \\
\hline \multirow[t]{3}{*}{5} & Left & - & $\sqrt{ }$ & - & - & $\sqrt{ }$ & $\sqrt{ }$ & - & $\sqrt{ }$ & - & $\sqrt{ }$ \\
\hline & Right & - & - & $\sqrt{ }$ & $\sqrt{ }$ & - & $\sqrt{ }$ & - & $\sqrt{ }$ & $\sqrt{ }$ & $\sqrt{ }$ \\
\hline & Percentage & $30 \%$ & $40 \%$ & $20 \%$ & $70 \%$ & $20 \%$ & $70 \%$ & $20 \%$ & $90 \%$ & $40 \%$ & $90 \%$ \\
\hline
\end{tabular}

$\mathrm{T} 12$ = T12 transverse process level $(\mathrm{TP}) ; \mathrm{L} 1=\mathrm{L} 1$ or between L1-L2 transverse processes $(\mathrm{TP}) ; \mathrm{L} 2=\mathrm{L} 2$ or betweenL2-L3 transverse processes (TP); L4 = crest of ilium at L4-TP. level; L5 = crest of ilium at L5-TP level; PF = psoas fascia; PVS = paravertebral space; QLF = quadratus lumborum fascia; TAP $=$ transversus abdominis plane; TFP $=$ transversalis fascia plane

injection was confirmed to be at the L3 lumbar transverse process in all the specimens as noted by the staining of the different tissue planes on the needle entry path. In nine of the ten (90\%) QLM injections, the dye was found to be ventral to the quadratus lumborum-transversalis fascia but dorsal to the endoabdominal fascia (Table 2). After one of the injections $(n=1 / 10)$, although the needle tip was seen to be ventral to the QLM, the injectate was not seen ventral to the QLM fascia on dissection, and the entirety of the dye was contained within the fascia ventral to the QLM. This was considered a failed TQL since no staining of lumbar nerves or medio-lateral spread was noted. The cephalad spread of the injectate ventral to the QLM fascia was up to the T12 transverse process in $30 \%(n=3 / 10)$, up to $\mathrm{L} 1$ in $40 \%(n=4 / 10)$, and up to L2 in 20\% $(n=2 / 10)$ (Fig. 2a). The injection with all the injectate within the muscle showed traces of the dye around the L3 level. The caudal extent of the dye spread in all $9 / 10$ successful injections was till the origin of the QLM from the inner lip of the iliac crest.

Medially the dye spread onto the lateral part of the psoas fascia and posteriorly to the origin of the psoas major muscle from the lumbar transverse processes in $70 \%(n=$ 7/10) of the injections (Fig. 2b). We did not detect any dye within the psoas major or on the lumbar plexus. In two injections (20\%), the medial extent of injectate spread extended to the lateral part of the thoracic paravertebral space (T11-12) with no clear cranio-caudal spread demonstrable within the space. A consistent spread of injectate was seen laterally towards the transversalis fascia in all the successful injections $(n=9 / 10)$ but a further spread into the TAP was seen in only four injections (Fig. 2). In nine of the ten specimens, a direct staining of the ilioinguinal and iliohypogastric (L1) nerves was observed ventral to the QLM (Fig. 3) while the subcostal nerve (T12) was additionally stained in six of the ten specimens (Table 2). The lateral femoral cutaneous nerve (L2 and L3) was stained in two of the nine successful injections as the nerve exited the psoas major muscle near the iliac crest and before it entered the fascia iliaca. Laterally, the T9-T12 nerves were stained in the transversalis fascia plane $(n=9 / 10)$ and the TAP $(n=4 /$ 10) with an overall cover of the T9-L3 dermatomes $(n=9 /$ 10). No intraperitoneal dye spread was observed.

\section{Case series}

The patient characteristics, dermatomal coverage, and analgesic parameters are presented in Table 3. The QLM and transverse process of $\mathrm{T} 3$ were visualized consistently in all patients. Two of the four $(50 \%)$ patients included for the clinical correlation had chronic pain preoperatively and were opioid tolerant. On assessment of the sensory blockade at $30 \mathrm{~min}$ following the block, all patients had a good dermatomal cover extending between T9/10-L1/2 (Fig. 4 and Table 3). The antero-posterior extent of the sensory block in all four patients extended from the anterior axillary line to mid-scapular line. The sensory blockade correlated with the staining of thoraco-lumbar nerves seen in the anatomical component of the study. No thoracic paravertebral spread of the sensory block was observed. None of the patients had any sensory/motor 


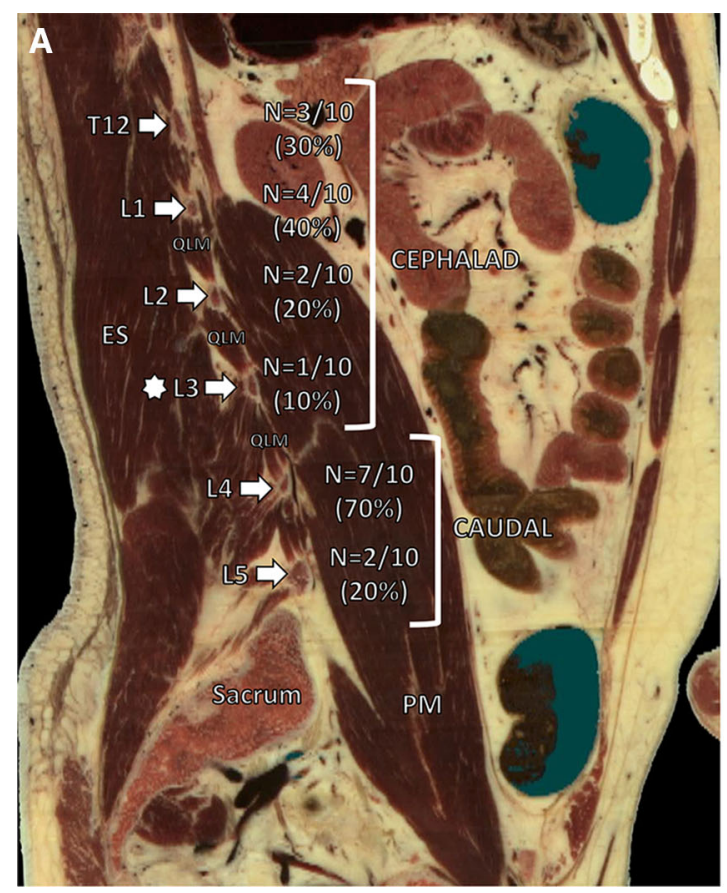

Fig. 2 Schematic anatomical slices representation of the (A) craniocaudal and (B) medio-lateral extent of dye spread following transmuscular quadratus lumborum injections performed at the level of L3 transverse process. * White asterisk indicates the point of injection of the study. + White cross indicates the injection site with the approach used by Ahikary et al. ${ }^{17}$ Carline et al., ${ }^{16}$ and Dam et al. ${ }^{18}$ The extent of spread has been presented in number (percentages). ES = erector spinae group of muscles; T12, L1, L2, L3, L4, and L5 denote the transverse process of the T12-L5 vertebra; $\mathrm{PM}=$ psoas major muscle;

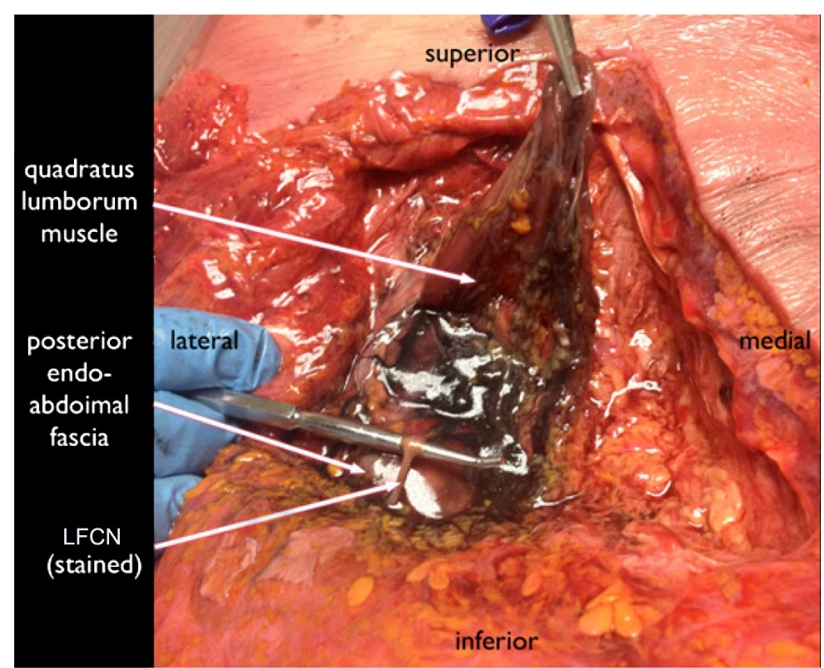

Fig. 3 A cadaveric specimen revealing direct staining of the lumbar nerve and spread anterior to the quadratus lumborum (reflected)

blockade in the femoral or obturator nerve distribution. We did not test for the sensation in the genitofemoral nerve distribution.

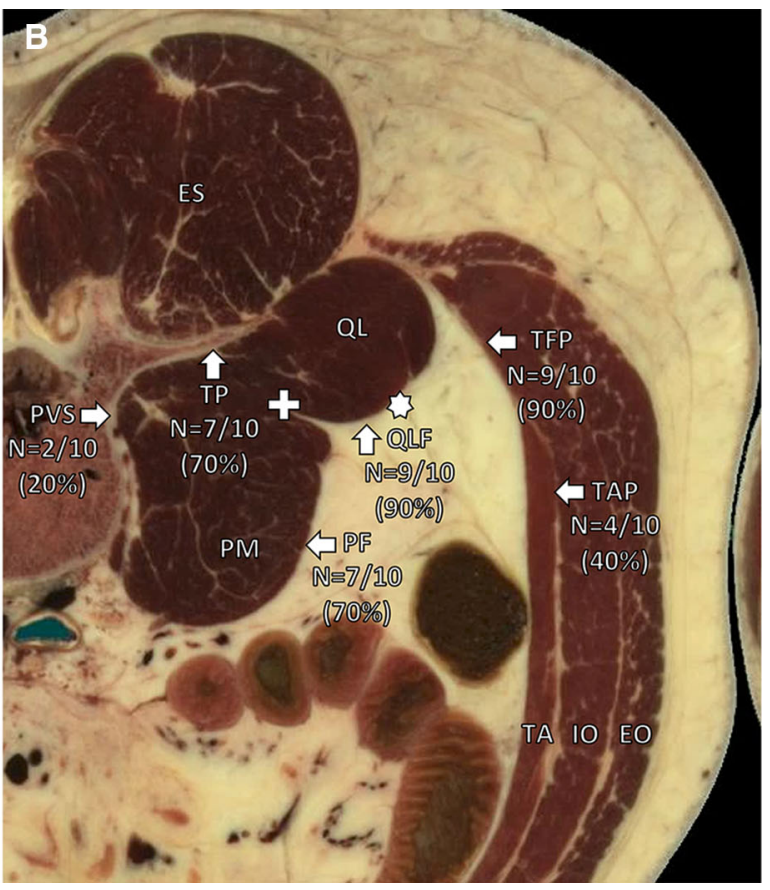

$\mathrm{QL} / \mathrm{QLM}=$ quadratus lumborum; $\mathrm{PVS}=$ paravertebral space; $\mathrm{TP}=$ transverse process; TFP $=$ transversalis fascia plane; TAP $=$ transversus abdominis plane; $\mathrm{TA}=$ transversus abdominis; $\mathrm{IO}=$ internal oblique; $\mathrm{EO}=$ external oblique; $\mathrm{TLF} / \mathrm{QLM}=$ quadratus lumborum fascia; $\mathrm{PF}=$ psoas fascia. Reproduced with permission from EPFL, Visible Human Visualization Software, http:// visiblehuman.epfl.ch and Gold Standard Multimedia (GSM) http:// www.gsm.org)

One patient (patient 1) who underwent a tibial nail exchange for non-union of a tibial fracture did not receive any other regional blocks apart from TQL, whereas all the other patients received popliteal sciatic and saphenous nerve blocks in addition to TQL. Both patients with chronic pain (patients 2 and 4) requested the TQL infusion to be restarted on the second postoperative day because of excruciating pain after discontinuation of the local anesthetic infusion for TQL. All patients were satisfied with the analgesia provided from TQL and all patients noticed when TQL had resolved. None of these patients had leakage or secondary block failure of the quadratus lumborum catheters. All patients were hemodynamically stable for the duration of the block infusion and had no evidence of spread of local anesthetic to other dermatomal territories. No complications were seen in any of the patients.

\section{Discussion}

Our study demonstrated the initial injectate spread following TQL injections and its clinical correlations. 

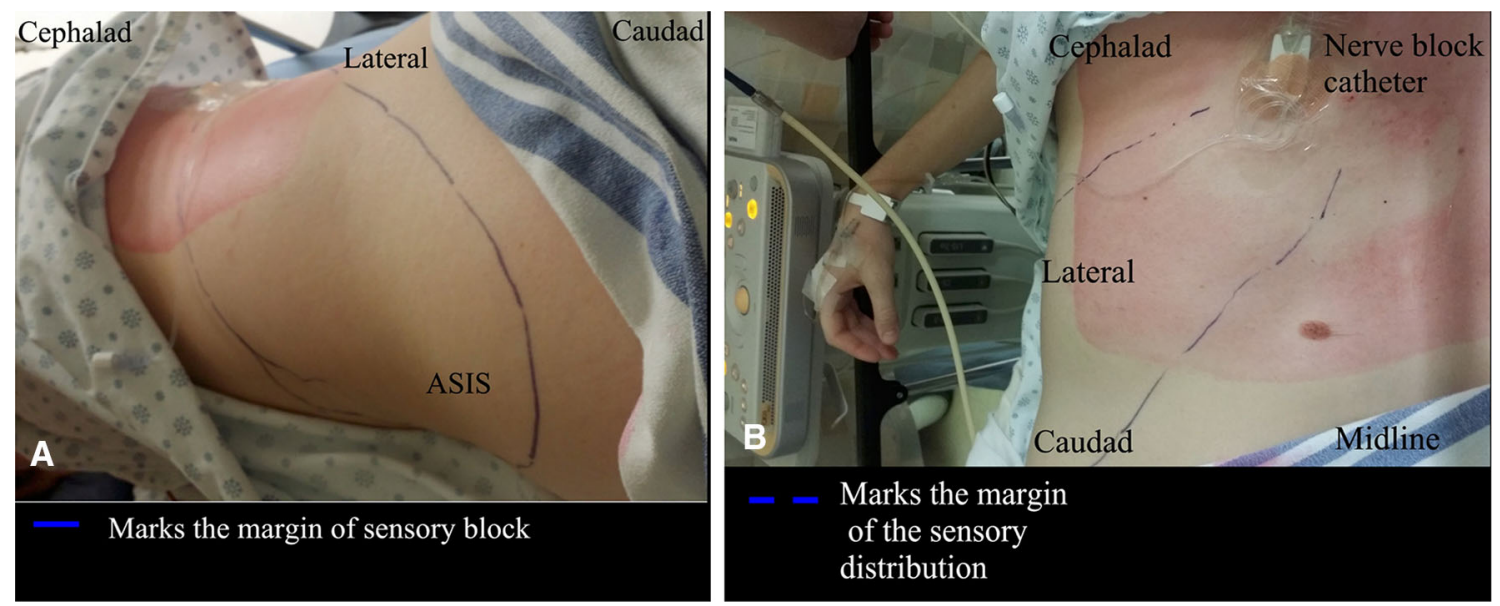

Fig. 4 The sensory distribution on the (A) anterolateral side and (B) back of a patient lying in a right lateral decubitus position following a transmuscular quadratus lumborum block

Quadratus lumborum block also provided effective analgesia following AIC bone graft in our case series. The injectate spread was consistent along the ventral surface of the muscle and stained the branches of T12-L2 nerves traversing the ventral surface of the QLM fascia. Both anatomical and clinical components of the study showed a consistent spread of injectate to cover the lower abdominal dermatomes (T9-L2) passing in close relation to the QLM and the TFP. The higher dermatomal cover in the anatomical component of the study was mainly due to the lateral spread of injectate rather than to a medial spread of injectate. In our study, the spread of injectate into the lateral part of the thoracic paravertebral space (T11-12) only occurred in $2 / 10$ cadavers. Furthermore, none of the patients experienced sensory blockade consistent with thoracic paravertebral spread of the local anesthetic following TQL.

The iliac crest is considered the gold standard for autologous bone grafts because of its large volume of cortical and cancellous bone. ${ }^{10}$ The iliac crest is divided into anterior (ventral) and posterior (dorsal) segments, ${ }^{7}$ both of which are accessed for bone grafts, but given the ease of access, the anterior iliac crest is the common site of bone graft retrieval. The periosteum of the iliac crest derives its rich innervation from the local nerves supplying the muscles attached to it ${ }^{6,7}$; hence, an understanding of the

Table 3 Case series

\begin{tabular}{|c|c|c|c|c|}
\hline Patient & Case 1 & Case 2 & Case 3 & Case 4 \\
\hline Age $(y r)$ & 21 & 29 & 37 & 40 \\
\hline Sex & Male & Female & Male & Female \\
\hline BMI & 26.1 & 20.3 & 28.2 & 23.5 \\
\hline Surgery & $\begin{array}{l}\text { AICBG for tibial nail } \\
\text { replacement }\end{array}$ & $\begin{array}{l}\text { AICBG for ankle } \\
\text { fusion }\end{array}$ & $\begin{array}{l}\text { AICBG for subtalar } \\
\text { fusion }\end{array}$ & $\begin{array}{l}\text { AICBG for subtalar } \\
\text { fusion }\end{array}$ \\
\hline Visualization of QLM structures & $\begin{array}{l}\text { QLM, TAP, TFP, and } \mathrm{L} 3 \\
\text { transverse process }\end{array}$ & $\begin{array}{l}\text { QLM and L3 } \\
\text { transverse process }\end{array}$ & $\begin{array}{l}\text { QLM and L3 } \\
\text { transverse process }\end{array}$ & $\begin{array}{l}\text { QLM TFP and L3 } \\
\text { transverse process }\end{array}$ \\
\hline Sensory level (30 min post block) & T10-L1 & T9/10-L1 & T8/9-L2 & T9-L1 \\
\hline Preoperative pain scores & 0 & 10 & 0 & 7 \\
\hline $24 \mathrm{hr}$ dynamic pain scores (NRS 0-10) & 5 & 8 & 1 & 7 \\
\hline $\begin{array}{l}\text { Preoperative analgesia consumption (oral } \\
\text { morphine equivalent) }\end{array}$ & 0 & 0 & 0 & 30 \\
\hline $\begin{array}{l}\text { PACU narcotic consumption (intravenous } \\
\text { morphine equivalents) }\end{array}$ & 6 & 3 & 0 & 3 \\
\hline $\begin{array}{l}\text { POD-0 narcotic consumption (oral } \\
\text { morphine equivalents) }\end{array}$ & 120 & 130 & 0 & 15 \\
\hline $\begin{array}{l}\text { POD-1 narcotic consumption (oral } \\
\text { morphine equivalents) }\end{array}$ & 240 & 230 & 60 & 31 \\
\hline
\end{tabular}

AICBG $=$ anterior iliac crest bone graft BMI = body mass index $;$ NRS $=$ numerical rating score PACU = post-anesthesia care unit; POD = postoperative day; TAP = transversus abdominis plane; TFP = transversalis fascia plane; QLM = quadratus lumborum muscle 
muscular attachments to the iliac crest is necessary to ensure adequate analgesia. The outer lip of the AIC provides attachment to the iliotibial band, tensor fascia lata, and lower fibres of the external oblique as well as latissimus dorsi muscles. The intermediate part of the iliac crest allows attachment to the internal oblique muscle while the transversus abdominis muscle attaches to the inner lip of the anterior iliac crest. Hence, the relevant innervation to the AIC is the lateral femoral cutaneous nerve (LFCN) of the thigh (supplies the tensor fascia lata and iliotibial band), ilioinguinal, and iliohypogastric nerve (supplying the lower fibres of the transversus abdominis, internal oblique), as well as the subcostal nerve (supplying the lower fibres of the external oblique). Both the transversalis fascia plane block (TFP) ${ }^{11}$ and $\mathrm{TAP}^{12}$ block have been utilized for analgesia following AIC bone graft with promising results, but the evidence for their use comes from a small retrospective study and a prospective open label study. While the TFP and TAP have the same target of ilioinguinal, iliohypogastric, and subcostal nerves, the basis for covering these nerves before they give off the lateral cutaneous branches is by a medial spread of the drug underneath the QLM. ${ }^{13}$ Future prospective studies may better delineate the differences in analgesic utility of TQL and TFP for AIC bone graft.

The quadratus lumborum, a small accessory muscle of inspiration located in the lower back, takes its origin from the internal lip of the posterior segment of the iliac crest and the adjoining iliolumbar ligament. It then inserts into the lower border of the twelfth rib and the tips of the transverse processes of the upper four lumbar vertebrae. The quadratus lumborum and erector spinae muscles divide the thoraco-lumbar fascia into three layers. The layer posterior to the erector spinae group of muscles is known as the posterior thoraco-lumbar fascia, the layer intervening between the erector spinae and the QLM is known as the middle thoraco-lumbar fascia, and the layer anterior to the quadratus lumborum is known as the transversalis fascia, which is in continuity with the transversalis fascia of the abdomen laterally, the psoas fascia medially, and the fascia iliaca inferiorly. ${ }^{14}$ The transversalis fascia covering the quadratus lumborum is traversed by the iliohypogastic (L1) and ilioinguinal (L1) nerves, both of which were consistently stained with the injectate in the cadaveric specimens following the TQL injections. The subcostal nerve (T12), which traverses the upper part of the QLM behind the kidney and arcuate ligament, was also covered in a majority of the specimens. The LFCN (L2,3) was stained in two of the nine successful cadaveric injections when the nerve exited the psoas major muscle above the level of the iliac crest. While the LFCN may or may not course in the transversalis fascia after its exit from the psoas muscle ${ }^{15}$ given that the nerve travels in the common fascial compartment of the transversalis fascia and iliac fascia, whether this nerve can be consistently anesthetized with a higher volume needs to be explored.

Other anatomical studies on TQL have noted different dispersions of injectate than that of our study. In the two recent studies on TQL, Carline et al. ${ }^{16}$ and Adhikary et al. ${ }^{17}$ noted a consistent spread of the dye into the lumbar paravertebral space and staining of L1-3 nerve roots, while all of our cadaveric injections had a medial extent of the dye limited to the lateral part of the psoas fascia. The injectate did not spread into the psoas muscle in any of the cadaveric specimens and was limited by the origin of the posterior part of the psoas major. We did not notice any evidence of motor or sensory blockade in the femoral or obturator nerve territories in patients receiving TQL in our case series, which supports our anatomical findings. In a recent study, Dam et al. ${ }^{18}$ noted no spread of injectate into the psoas major muscle or the lumbar plexus, similar to our findings. In contrast to our study findings, Dam et al. ${ }^{18}$ noted a consistent spread of injectate into the thoracic paravertebral space with both the transmuscular QLM and the transverse-oblique approach to the transmuscular QLM. The differences in spread to the thoracic paravertebral space seen in our study compared to those by Dam et al., ${ }^{18}$ Carline et al., ${ }^{16}$ and Adhikary et al. ${ }^{17}$ may be attributed to the needle path and the final needle tip position used for their cadaveric injections. While we used a lateral-medial needle insertion with the intended target of the final needle tip to be on the ventral free edge of the QLM, Carline et al., ${ }^{16}$ Adhikary et al., ${ }^{17}$ and Dam et al. ${ }^{18}$ targeted the ventral surface of the QLM adjoining the psoas major muscle. (Fig. 2b) We speculate that the ventral and dorsal portions of the psoas major muscle may remain separated, ${ }^{19}$ thereby creating a path for the injectate to flow in between the two parts of the psoas major and thus the lumbar plexus as seen by Carline et al. ${ }^{16}$ and Adhikary et al. ${ }^{17}$ While Dam et al. ${ }^{18}$ utilized the approach similar to that of Carline et al. ${ }^{16}$ and Adhikary et al., ${ }^{17}$ the observed differences of the former study from the latter two studies may be partly due to the differences in the embalmed nature of the cadaveric specimens and the volume or properties of the injectate used in their studies. Nevertheless, further studies may be needed to examine the impact of these subtle differences in the final needle tip location in contributing to the variation of observed injectate spread pattern. The inter-fascial communication between the fascia of the thorax, abdomen, and pelvis has been described by several authors. ${ }^{20-23}$ Although an injectate of sufficient volume and pressure aimed towards the midline could theoretically track cephalad and medially, we did not see it following any of our injections. We hypothesize that the flow of injectate probably followed the path of least resistance and tracked 
more towards the TFP and the TAP rather than towards a tight fascial compartment within the psoas major medially.

A failure of the needle tip to penetrate through the quadratus lumborum fascia in one of the ten injections resulted in the injectate being deposited entirely within the muscle belly with a failure to stain any of the nerves traversing the QLM or TFP. Success of a lateral to medial approach for TQL block most likely hinges on penetrating through the anterior QLM fascia. Some of the limitations of the current study include the limited number of cases for the clinical evaluation and the possible variability in the injectate spread when the TQL is performed at a higher level or using a medial-lateral needle approach.

When considering other factors on the spread of the injectate, one of drawbacks of this study was that there were limited demographic data of the cadavers such as individual BMI being available for examining the influence of the body habitus on the extent of spread. Another potential limitation of this study was that the viscosity of the injectate used was more viscous than the standard local anesthetic solution. We chose a more viscous solution to limit the dye diffusion across the tissues in an attempt to allow accurate identification of tissue planes and to estimate conservatively the spread of injectate. However, this could have contributed to underestimating the pattern of injectate spread seen in our cadavers.

In summary, a lateral-medial approach for TQL reliably covers the T9-L2 dermatomes and consistently covers the ilioinguinal, iliohypogastric, and subcostal nerves while an inconsistent spread was seen towards the lateral femoral cutaneous nerve. Injectate spreads along the transversalis fascia over the QLM and laterally into the TFP and TAP. A reliable paravertebral spread could not be established in either the anatomical or clinical component of the study. Preliminary data suggested that TQL could provide effective analgesia following an AIC bone graft in our case series, albeit with a limited patient number. However, it is important to point out that since the clinical part of the study involved only descriptive observation of four patients, the results cannot be generalized. Further randomized controlled trials are needed to establish the role of TQL in comparison to other analgesic modalities in surgeries involving the lower abdomen, iliac crest, and hip.

Conflict of interest Dr. Ban Tsui, patent licensing agreement on ECath with Pajunk.

Editorial responsibility This submission was handled by Dr. Philip M. Jones, Associate Editor, Canadian Journal of Anesthesia.

Author Contributions Rakesh Sondekoppam contributed to the conception, study design, performance of blocks, dissection recording, manuscript proof read, and suggestion and approved the final manuscript. Rakesh Sondekoppam is the archival author. Vivian Ip contributed to the case series and manuscript preparation and approved the final manuscript. David Johnston contributed to the performance of blocks, dissection recording, and manuscript composition and approved the final manuscript. Vishal Uppal contributed to the study design, performance of blocks, manuscript proof read, and suggestion and approved the final manuscript. Marjorie Johnson contributed to the dissection of cadavers and direct description and recording of ink spread and approved the final manuscript. Sugantha Ganapathy oversaw the project and manuscript input and approved the final manuscript. Ban C.H. Tsui oversaw the project, manuscript preparation, and conduct of case series and approved the final manuscript. All authors attest to the integrity of the original data and the analysis reported in this manuscript.

Funding None.

\section{References}

1. Carson M. Anterior iliac crest bone graft. In: Haggerty CJ, Laughlin RM, editors. Atlas of Operative Oral and Maxillofacial Surgery. Hoboken, NJ, USA: John Wiley \& Sons, Inc.; 2015 .

2. Barone A, Ricci M, Mangano F, Covani U. Morbidity associated with iliac crest harvesting in the treatment of maxillary and mandibular atrophies: a 10-year analysis. J Oral Maxillofac Surg 2011; 69: 2298-304.

3. Shin SR, Tornetta P 3rd. Donor site morbidity after anterior iliac bone graft harvesting. J Orthop Trauma 2016; 30: 340-3.

4. Silber JS, Anderson DG, Daffner SD, et al. Donor site morbidity after anterior iliac crest bone harvest for single-level anterior cervical discectomy and fusion. Spine (Phila Pa 1976) 2003; 28: 134-9.

5. Visser EJ. Chronic post-surgical pain: epidemiology and clinical implications for acute pain management. Acute Pain 2006; 8: 7381.

6. Howard MA, Dickie SR. Chapter 9: Comprehensive trunk anatomy. In: Neligan PC, editor. Plastic Surgery. 3rd ed. NY: Elsevier Health; 2013. p. 220-38.

7. Birch R. Chapter 80: Pelvic girdle, gluteal region and thigh. In: Standring S, editor. Gray's Anatomy: The Anatomical Basis of Clinical Practice. 41st ed. London: Elsevier; 2016. p. 1337-75.

8. Elsharkawy H. Quadratus lumborum block with paramedian sagittal oblique (subcostal) approach. Anaesthesia 2016; 71: 2412.

9. Børglum J, Moriggl B, Jensen $K$, et al. Ultrasound-guided transmuscular quadratus lumborum blockade. Br J Anaesth 2013; 111. DOI: https://doi.org/10.1093/bja/el_9919.

10. Giannoudis PV, Karadimas EJ, Kanakaris NK. Anterior iliac crest bone graft harvesting. In: Giannoudis PV, editor. Practical Procedures in Orthopedic Surgery. London: Springer Inc.; 2012. p. 51-5.

11. Chin KJ, Chan V, Hebbard P, Tan JS, Harris M, Factor D. Ultrasound-guided transversalis fascia plane block provides analgesia for anterior iliac crest bone graft harvesting. Can J Anesth 2012; 59: 122-3.

12. Chiono J, Bernard N, Bringuier $S$, et al. The ultrasound-guided transversus abdominis plane block for anterior iliac crest bone graft postoperative pain relief: a prospective descriptive study. Reg Anesth Pain Med 2010; 35: 520-4.

13. Hebbard $P D$. Transversalis fascia plane block, a novel ultrasound-guided abdominal wall nerve block. Can J Anesth 2009; 56: 618-20.

14. Willard FH, Vleeming A, Schuenke MD, Danneels L, Schleip R. The thoracolumbar fascia: anatomy, function and clinical considerations. J Anat 2012; 221: 507-36. 
15. Üzel M, Akkin SM, Tanyeli E, Koebke J. Relationships of the lateral femoral cutaneous nerve to bony landmarks. Clin Orthop Relat Res 2011; 469: 2605-11.

16. Carline L, McLeod GA, Lamb C. A cadaver study comparing spread of dye and nerve involvement after three different quadratus lumborum blocks. Br J Anaesth 2016; 117: 387-94.

17. Adhikary SD, El-Boghdadly $K$, Nasralah Z, Sarwani N, Nixon $A M$, Chin KJ. A radiologic and anatomic assessment of injectate spread following transmuscular quadratus lumborum block in cadavers. Anaesthesia 2017; 72: 73-9.

18. Dam M, Moriggl B, Hansen CK, Hoermann R, Bendtsen TF, Børglum $J$. The pathway of injectate spread with the transmuscular quadratus lumborum block: a cadaver study. Anesth Analg 2017; 125: 303-12.

19. Farny J, Drolet P, Girard M. Anatomy of the posterior approach to the lumbar plexus block. Can J Anaesth 1994; 41: 480-5.
20. Saito T, Den S, Tanuma K, Tanuma Y, Carney E, Carlsson C. Anatomical bases for paravertebral anesthetic block: fluid communication between the thoracic and lumbar paravertebral regions. Surg Radiol Anat 1999; 21: 359-63.

21. Saito T, Gallagher ET, Cutler S, et al. Extended unilateral anesthesia. New technique or paravertebral anesthesia? Reg Anesth 1996; 21: 304-7.

22. Karmakar $M K$, Chung DC. Variability of a thoracic paravertebral block. Are we ignoring the endothoracic fascia? Reg Anesth Pain Med 2000; 25: 325-7.

23. Cheema $S$, Ilsley $D$, Richardson $J$, Sabanathan $S . \quad$ A thermographic study of paraveterbral analgesia. Anaesthesia 1995; 50: 118-21. 